\title{
Intraductal oncocytic papillary neoplasm: A rare pancreatic mass
}

\author{
Mayur Brahmania MD, Darshan Khangura MD, Michael Cantor MD
}

\section{CASE PRESENTATION}

A previously healthy 45 -year-old Caucasian woman presented with a six-year history of intermittent nausea and vomiting. Physical examination was unremarkable. Screening laboratory work, including complete blood count, electrolytes, liver function tests, thyroid stimulating hormone, cortisol and hemoglobin A1C, were within normal limits. An esophagogastroduodenoscopy revealed fundic gland polyps with no evidence of dysplasia, Helicobacter pylori or celiac disease. Given the ongoing symptoms, an ultrasound of the abdomen and, subsequently, a computed tomography $(\mathrm{CT})$ scan were performed revealing a $3.8 \mathrm{~cm} \times$ $4.5 \mathrm{~cm}$ cystic mass at the junction of the head and body of the pancreas as well as a $1 \mathrm{~cm}$ cystic lesion in the head of the pancreas. Pancreatic

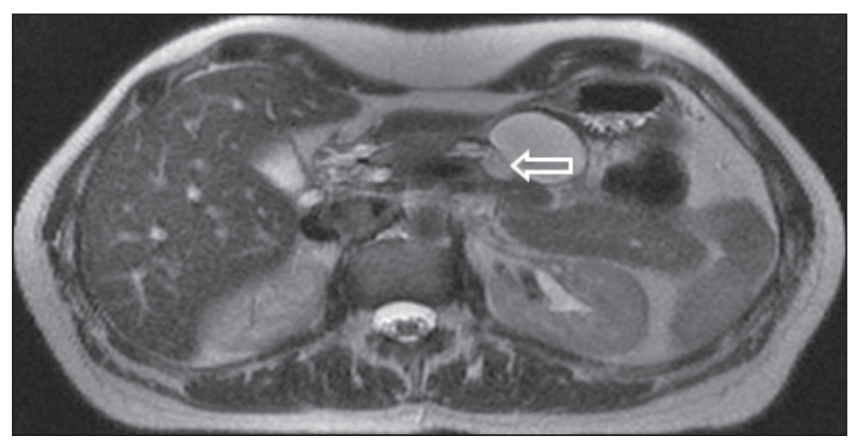

Figure 1) Magnetic resonance imaging showing a $2.7 \mathrm{~cm} \times 3.9 \mathrm{~cm}$ lesion in the head and $5.0 \mathrm{~cm} \times 5.2 \mathrm{~cm}$ in the distal pancreatic body (arrow)

magnetic resonance imaging (MRI) and magnetic resonance cholangiopancreatography (MRCP) demonstrated a lobular unilocular $5 \mathrm{~cm} \times 3 \mathrm{~cm}$ cystic lesion in the body of the pancreas that appeared to directly communicate with the main pancreatic duct (Figure 1). Endoscopic ultrasound confirmed a $2.7 \mathrm{~cm} \times 3.9 \mathrm{~cm}$ cystic lesion in the neck of the pancreas with two small mural nodules measuring up to $7 \mathrm{~mm}$ (Figure 2). Fine-needle aspiration revealed no malignant cells on cytology; however, cyst fluid analysis showed a lipase level of $13,739 \mathrm{U} / \mathrm{L}$, carcinoembryonic antigen (CEA) level of $2.09 \mu \mathrm{g} / \mathrm{L}$ and an amylase level of 6,330 U/L. Given the dimensions of the tumour and discrepancy of CEA and lipase levels, a surgical opinion was obtained entertaining the possibility of a side branch intraductal papillary mucinous neoplasm (IPMN). A subtotal pancreatectomy and splenectomy was subsequently performed. On gross description, sectioning of the cystic lesion showed multiloculation with a smooth inner surface and filling with clear mucinous cells. Microscopically, architecture showed a complex multilayer with focal arborizing papillae with no duct communication. Cytologically, most of the cells showed an oncocytic appearance with enlarged nuclei, prominent nucleoli and scattered mitotic figures (Figure 3). These features are consistent with an intraductal oncocytic papillary neoplasm (IOPN) with high-grade dysplasia. No adjuvant radiation or chemotherapy was performed. Following resection, symptom resolution and follow-up MRI did not show any local recurrent disease.

\section{DISCUSSION}

IPMNs are mucin-producing pancreatic cystic neoplasms involving the main pancreatic duct and/or its major side branches. Histologically, IPMNs are divided into four distinct subtypes: gastric, intestinal, pancreatobiliary and oncocytic (1). IOPN is a rare subtype of IPMN, the clinical and diagnostic features of which remain unclear. In 1996, Adsay et al (2) first described this neoplasm as a distinct cystic intraductal tumour with oncocytic features and mucin production. Data regarding the accuracy of preoperative imaging and natural history of these lesions are limited. Similar to many pancreatic cystic neoplasms, no single imaging method is able to accurately diagnose IOPN. MRI and MRCP can assist with the diagnosis in their ability to demonstrate main pancreatic duct communication, which occurs in most but not all cases of IOPN (3). Although MRI may also provide enhanced morphological detail, subtle features, such as mural nodularity, may be missed. As in our case, endoscopic ultrasound may provide superior imaging detail of the cyst architecture, which can upstage the lesion and alert the clinician that a more sinister pathology is present. Unfortunately, cyst fluid CEA and cytology can be inaccurate. Identification of additional cyst fluid markers is needed to enhance diagnostic accuracy. Further investigation of this rare neoplasm will help characterize its clinical features, diagnosis, treatment and prognosis.

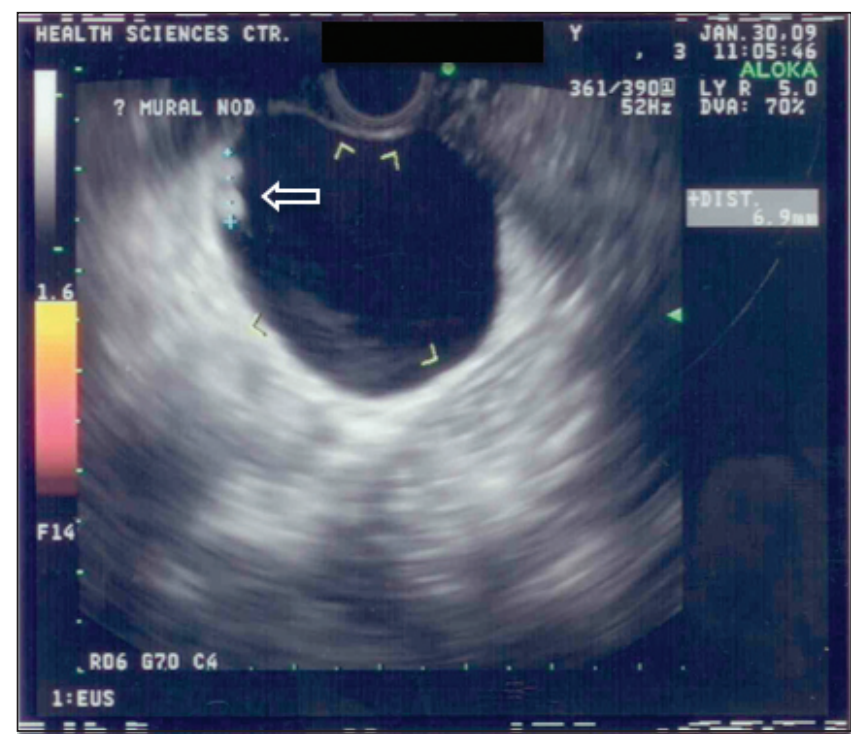

Figure 2) Endoscopic ultrasound showing mural nodules (arrow)

Department of Medicine, Division of Gastroenterology, Health Sciences Hospital, University of Manitoba, Winnipeg, Manitoba Correspondence: Dr Mayur Brahmania, Health Sciences Hospital, 804F-175 McDermot Avenue, Winnipeg, Manitoba R3E 3P4.

Telephone 204-789-3369, fax 204-789-3972, e-mail mbrahmania@gmail.com

Received for publication March 18, 2013. Accepted May 6, 2013 


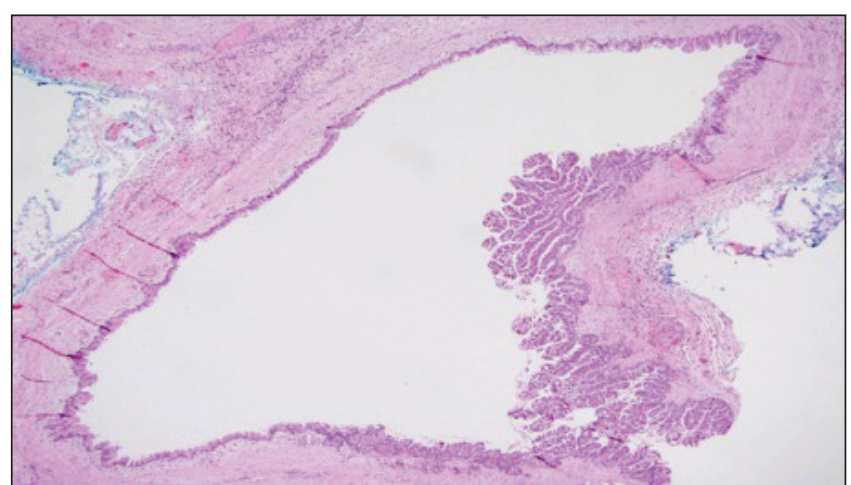

Figure 3) Hematoxylin and eosin stain showing complex papillary and micropapillary formation of oncocytic cells (high-power field, original magnification $\times 40$ )

\section{REFERENCES}

1. Al-Haddad M, Schmidt MC, Sandrasegaran K, Dewitt J. Diagnosis and treatment of cystic pancreatic tumors.

Clin Gastroenterol Hepatol 2011;9:635-48.

2. Adsay NV, Adair CF, Heffess CS, Klimstra DS. Intraductal oncocytic papillary neoplasms of the pancreas.

Am J Surg Pathol 1996;20:980-94.

3. Fischer MA, Donati O, Heinrich S, et al. Intraductal oncocytic papillary neoplasm of the pancreas: A radio-pathological case study. JOP J Pancreas (Online) 2010;11:49-54. 


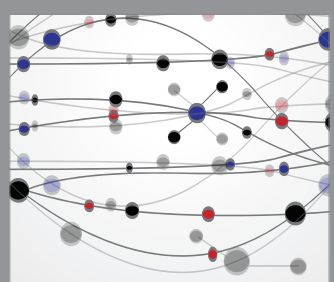

The Scientific World Journal
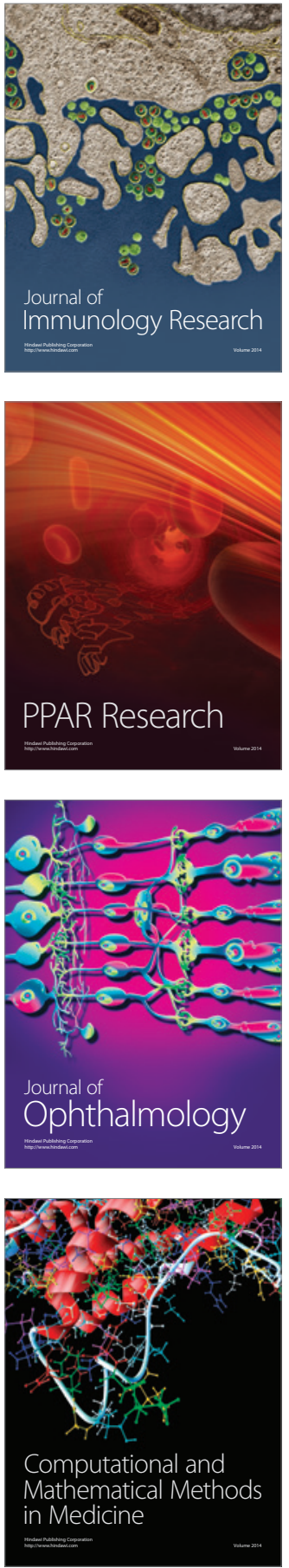

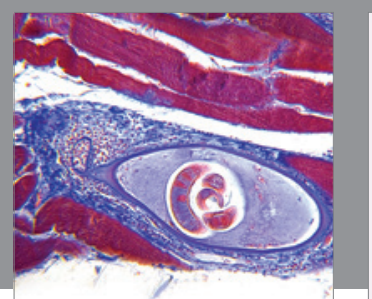

Gastroenterology Research and Practice

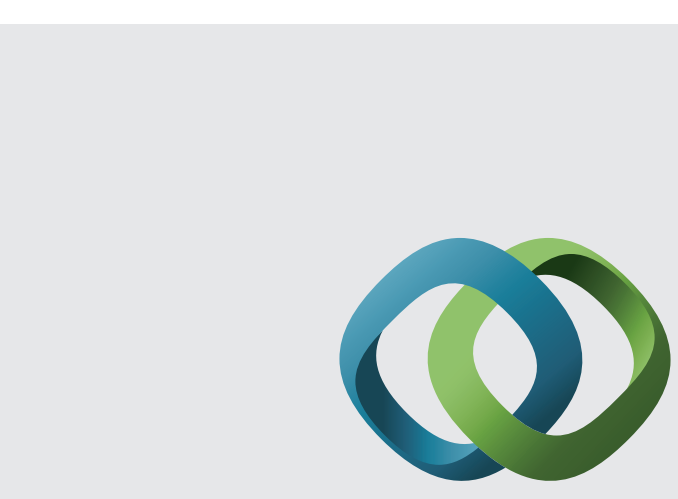

\section{Hindawi}

Submit your manuscripts at

http://www.hindawi.com
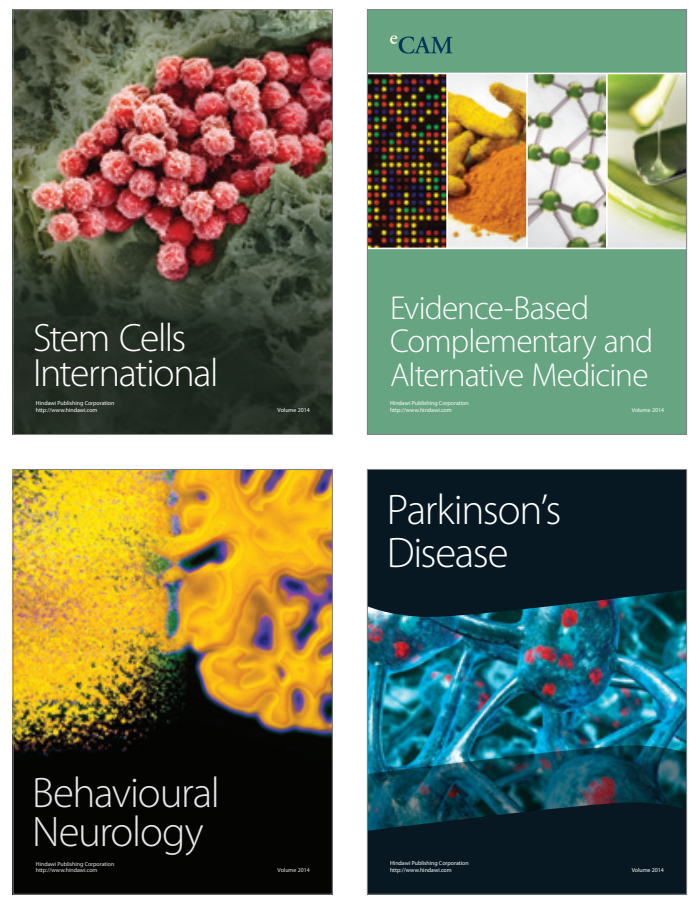
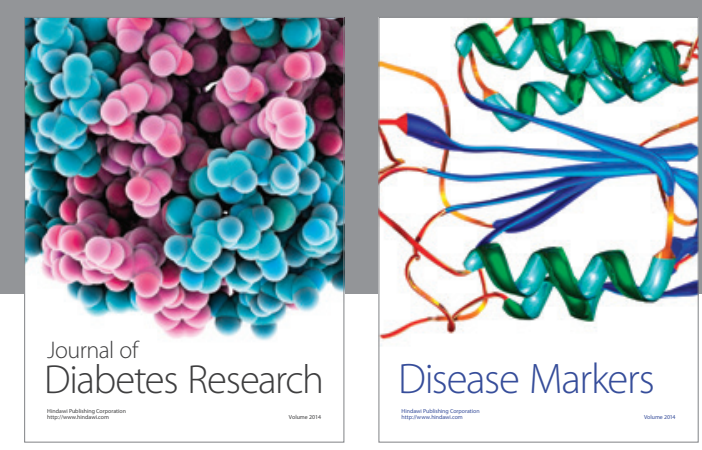

Disease Markers
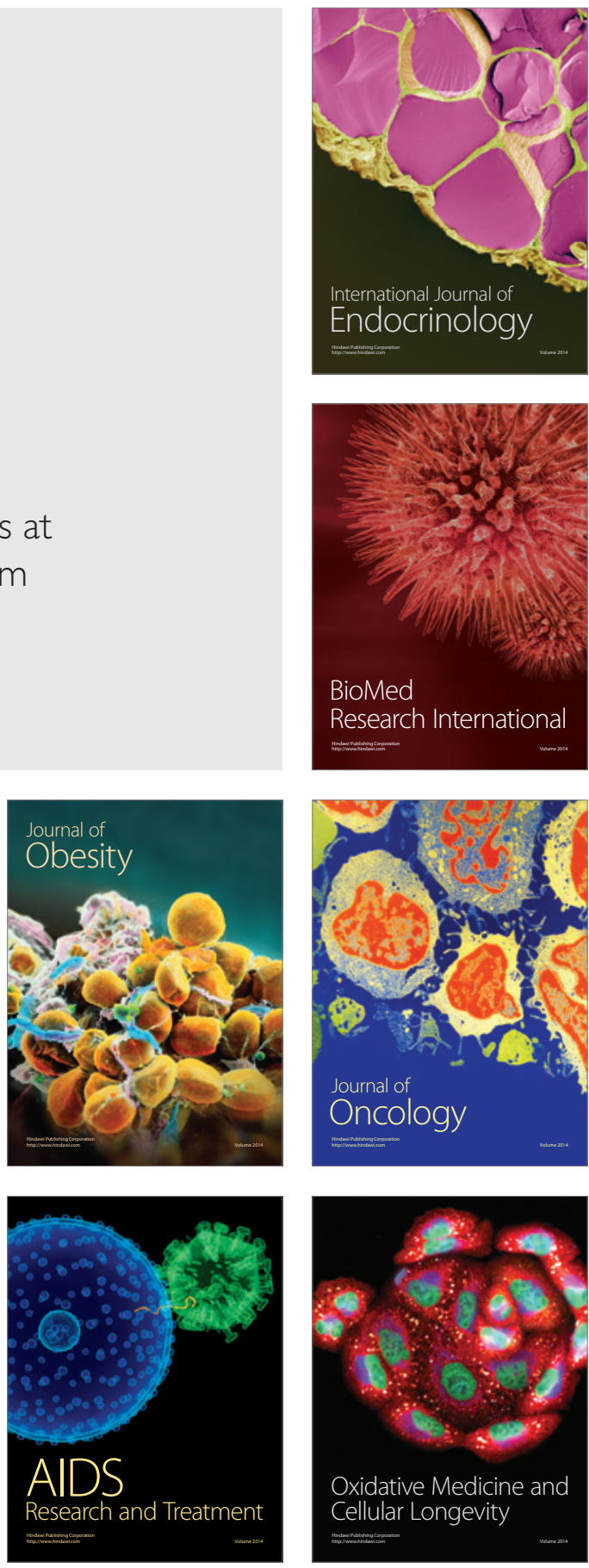